\title{
Die Erkennung der Monozyten im Differentialblutbild mit unterschiedlicher Technik
}

\author{
The Monocytes: Their Recognition by Different Techniques
}

\author{
H. J. Avenarius, J. Deinhardt
}

Aus der Abteilung Hämatologio-Onkologie (Dir.: Prof. Dr. H. Poliwoda) des Zentrums für Innere Medizin und Dermatologie der Medizinischen Hochschule Hannover

\begin{abstract}
Zusammenfassung:
Verglichen wurde die mikroskopische Untersuchung am handgefertigten Ausstrich, der noch immer gängigen Standardmethode, mit der automatischen Differenzierung eines Spinnerausstrichs einschließlich individuellem Review aller Monocyten (Microx 120), der Anfärbung und Auszählung Esterase-positiver Zellen im Ausstrich, der Analyse mit den monoclonalen Antikörpern CD 14 und 45 mit dem EPICS Profile II, dem Coulter ${ }^{\circ}$ STKS und schließlich dem Coulter ${ }^{\circledR}$ MAXM.

Es hat sich gezeigt, daß das Mikroskopieren eines handgefertigten Ausstrichs nicht als Beurteilungsmaßstab gelten kann. Beim Vergleich der visuellen Differenzierung von Spinnerausstrichen (Microx 120) mit dem MAXM zeigte sich eine Korrelation, die mit einem Korrelationskoeffizienten von $r=0,742$ bei einer Steigerung der Regressionsgeraden von 1,02 als befriedigend angesehen werden kann. Angesichts der grundsätzlichen Problematik einer Bestimmung der absoluten Monozytendichte im peripheren Blut wird das Ergebnis auch der "besten Zählmethode" stets nur in Verbindung mit dem Wissen des behandelnden Arztes über den klinischen Befund aussagekräftig sein.
\end{abstract}

\section{Schlüsselwörter:}

Monozyten-Zählung - Methodik

\section{Summary:}

The microscopic results of differentiation of blood smears made by hand, as yet the most common method in smaller laboratories, were compared with the automatical differentiation of a spined blood smear with individual control of all monocytes (Microx 120), the staining and counting of esterase-positive cells on a slide, the analysis by monoclonal antibodies CD 14 and 45 by EPICS Profile II, the Coulter ${ }^{\oplus}$ STKS, and finally the Coulter ${ }^{\oplus}$ MAXM.

It was turned out that the differentiation of a hand-made slide is not a criterion for correct analysis. The comparison of the optical review of spined smears (Microx 120) with the MAXM showed a correlation $r=0,742$ and a slope of 1,02 which may be regarded as satisfactory.

Facing the fundamental problem of recognising the density of monocytes in peripher blood, the result of even any "best method" may only be considered as valid in conjunction with the doctor's knowledge about his patients particular situation.

Keywords:

Monocyte count - methodics

\section{Einleitung}

Wegen der natürlichen Polymorphie der Monozyten stellt sich das Problem einer sicheren Erkennung schon im normalen Blutausstrich. Begemann und Rastetter (1) bieten in ihrem Atlas 15 unterschiedlich aussehende, jedoch àls normal zu differenzierende Abbildungen an. Alle anderen Zellen des peripheren Blutausstriches werden allenfalls mit 4 Abbildungen dargestelit. Jede Abbildung gibt Hilfestellung für das Mikroskopieren. Dabei besteht kein Zweifel daran, daß das, was unter dem Mikroskop zu sehen ist - in eine Ebene gedrückt und angefärbt - nicht dem Erscheinungsbild der.Zelle im dreidimensionalen Raum entspricht. Dieses wechselt während der Strömung im Blut und bei der Inanspruchnahme der jeweils spezifischen Funktion der Zelle. Auf dem Objektträger haben wir es immer mit Artefakten zu tun, die dadurch entste- hen, daß dreidimensionale Körper in zwei Dimensionen nicht abgebildet, sondern mehr oder weniger kräftig gedrückt werden. Dennoch haben sich für diese Artefakte Begriffe finden lassen, die eine Zuordnung erlauben.

Aus historischen Gründen gilt die mikroskopische Differenzierung (bei guter Ausbildung in dieser Disziplin) noch immer als Standard, an dem sich die immer zahlreicher auf dem Markt erscheinenden teil- und vollautomatischen Differenziergeräte zunächst messen lassen müssen. Die Begriffe, die vom mikroskopischen Bild abstrahiert wurden, werden übernommen, auch wenn die jeweilige Technik der Differenzierung eine andere Nomenklatur nahelegt. Beim Einsatz eines Durchflußcytometers werden die Zellen immer häufiger nach dem Antikörper benannt, mit dem sie positiv reagieren, wiederum aber nicht, ohne einen erklärenden Bezug zu den vertrauten Zellbildern herzustellen. 


\section{Diagnostin an Deurschland hat einen guten amen: Belnering}

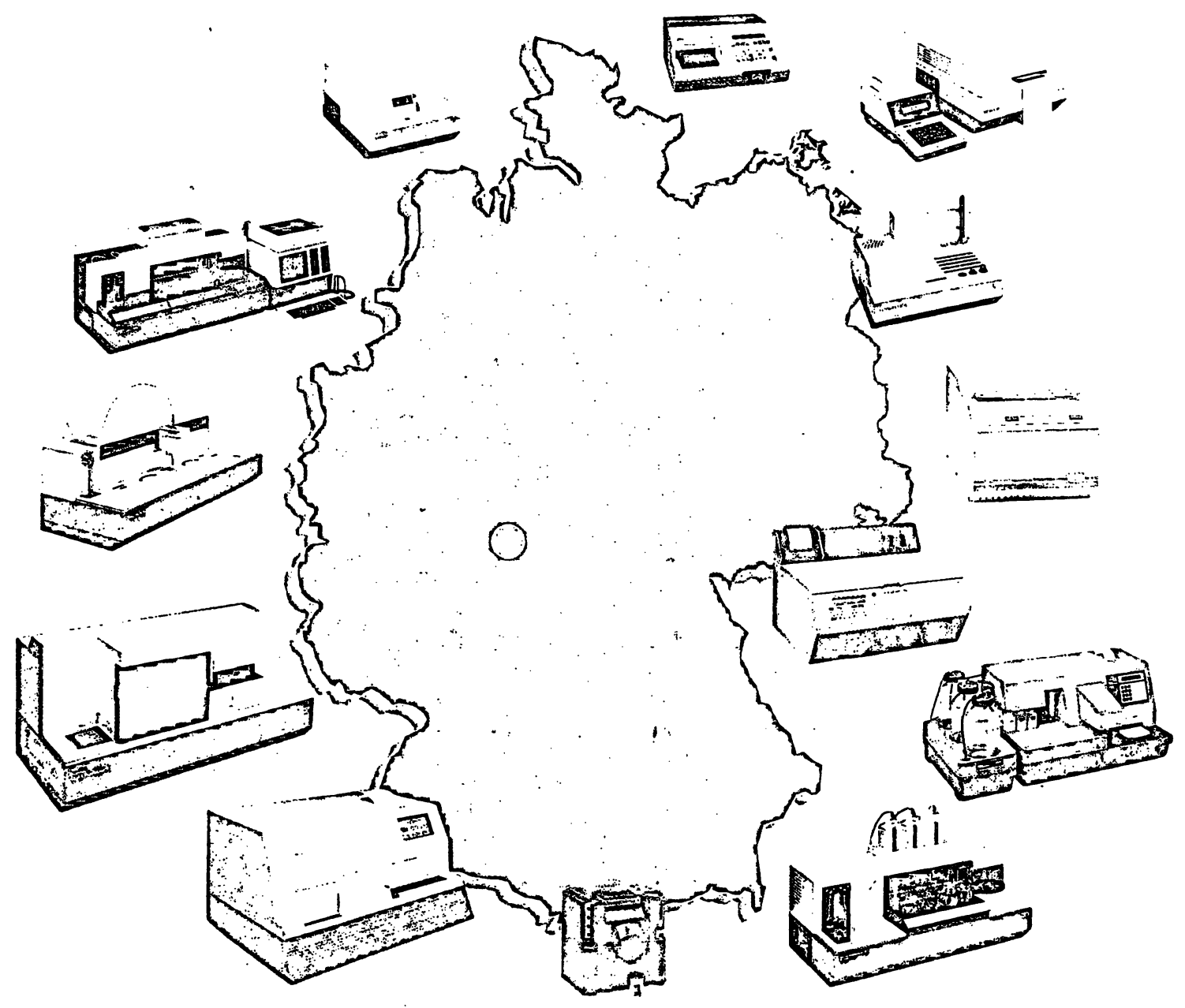

Behring bietet Innen ein umfassendes Diagnostika-Programm. Von qualitativen ScreeningTesten für die ärzlliche Praxis, die leicht zu handhaben und schnell abzulesen sind, bis zu hochspezifischen automatisierten Systemen für die quantitative Laboratoriumsdiagnostik.

Behring arbeitet intensiv an neuen Entwicklungen, die Ihre Probleme immer intelligenter und exakter lösen.

Sprechen Sie mit uns über 


\section{Stenerdienst für den Arzi}

Die für den Arzt einschlägigen Urteile finden Sie - nach Stichworten geordnet - in dem Loseblattwerk von Obersteuerrat Robert Linden.

Das Werk enthält u. a. die statistischen Kostensätze der Ärzte aller Fachrichtungen sowie Testbögen, die eine genaue Kostenanalyse der Praxis ermöglichen. Der Rationalisierung Ihrer Praxis dient das ausführliche „ABC der abzugsfähigen Ausgaben bei der Einkommensteuer".

\section{Hand aufs Herz}

* Verschenken Sie nicht auch Jahr für Jahr viel Geld an das Finanzamt?

* Nehmen Sie alle legalen Steuerminderungsmöglichkeiten in Anspruch?

* Stellen Sie rechtzeitig die notwendigen Anträge?

* Haben Sie Zeit und Gelegenheit, sich über Ihre berufsspezifischen Steuerfragen in den Steuerfachzeitschriften zu orientieren?

* Verlassen Sie sich ganz auf Ihren Berater?

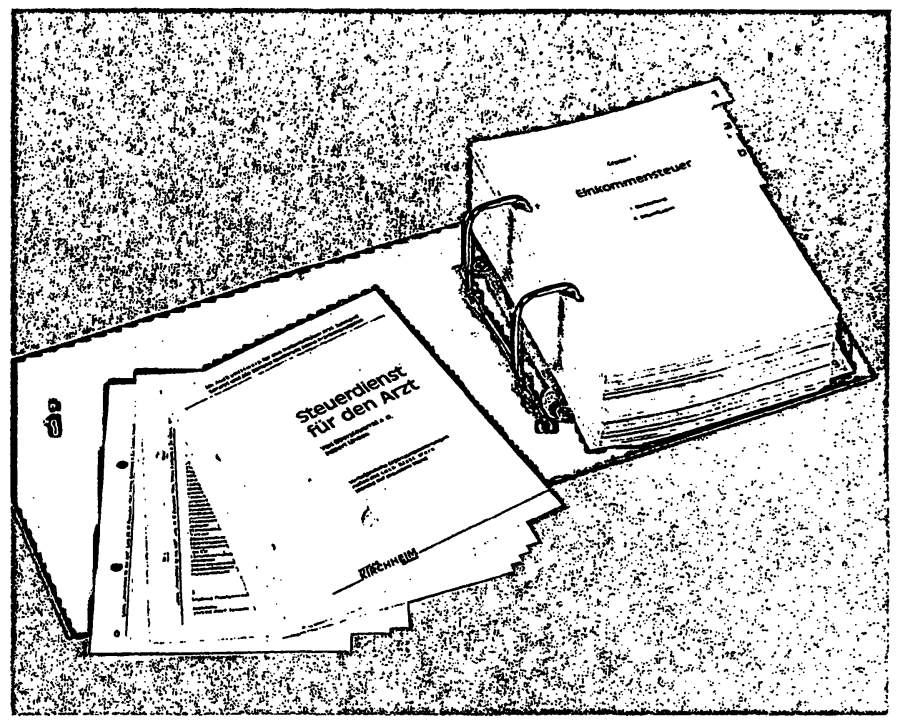

* Haben Sie schon einmal einen Praxiskostentest gemacht?

* Wissen Sie, ob Sie mehr oder weniger als Ihre vergleichbaren Kollegen verdienen?

\section{VERLAG}

\section{KIRCHHEIM Postfach 2524,6500 Mainz 1 \\ MAINZ}

Hiermit bestelle ich ein Crundwerk „Steuerdienst für den Arzt" zum Preis von 79,80 DM und die künftig vierteljährlich erscheinenden Ergänzungslieferungen zum Seitenpreis von -.37 DM.

Sie garantieren mir, daß ich diese Bestellung binnen einer Frist von einer Woche schriftlich widerrufen kann. Der Widerruf ist an den Verlag Kirchheim, Postfach 25 24, 6500 Mainz, zu richten. Zur Wahrung der Frist genügt die rechtzeitige Absendung des Widerrufs.

Datum/Unterschrift:
Anschrift:

Name:

Straße:

PLZ/Ort: 
Weil unterschiedliche neue Techniken entwickelt wurden, die miteinander und natürlich mit der Mikroskopiertechnik konkurrieren, versucht man an der Verbindlichkeit der herkömmlichen Begriffsbildung und Benennung festzuhalten, ob auf die Dauer zurecht, muß ernsthaft bezweifelt werden. Daß eine Verständigung auch bei unterschiedlicher Technik überhaupt möglich ist, hängt damit zusammen, daß die Analyse gleicher Blutproben mit unterschiedlichen Verfahren zumindest vergleichbare Verteilungsmuster aller differenzierten Zellen zeigt. Die durch die jeweilige Spezifität der Methode entstehenden Phänomene bleiben unübersetzbar.

Dem weniger Geübten fällt die Erkennung der Monozyten wegen deren Polymorphie schwer. Bei Geübten beträgt die Rate der Fehlzuordnung im Durchschnitt immerhin auch $9,1 \%$ (2). Wie steht es mit den automatisierten Analysegeräten, denen "Erfahrung" als Software schrittweise installiert wurde? Dieser Frage wurde von uns nachgegangen. Insbesonders zielte die vorliegende Untersuchung auf die Evaluation des neu auf dem Markt angebotenen Coulter ${ }^{\circledR}$ MAXM im Vergleich zur mikroskopischen Untersuchung.

\section{Methode}

An 210 Blutproben, die nach Passage des Coulter ${ }^{\circledR}$ STKS Monozytenwerte im Normbereich (3-14\%) zeigten, wurden parallel die im folgenden genannten Differenzierungsverfahren durchgeführt:

\subsection{Mikroskopische Verfahren}

2.1.1 Mit der Hand ausgestrichene Präparate wurden nach Wright gefärbt, danach von 2 Mitarbeitern je 100 Zellen, bei weniger als $5 \%$ Monozyten je 200 Zellen (statistische Absicherung der Ergebnisse) differenziert. Zieht man den Bluttropfen auf dem liegenden Objektträger hinter einem Deckglas her über das Feld, so kann es dazu kommen, daß die kleineren Zellen schließlich überwiegend in der Mitte des Feldes, die größeren, gerade auch die Monozyten, dagegen am Rande liegen. Hier soll das Durchmustern der Ausstriche in der "Määnderform" einen gewissen Ausgleich schaffen. Diese Ausstrichtechnik, die im eigenen Labor seit Jahren-nicht mehr eingesetzt wird, wurde hier gewählt, weil die optimale „Spinner-Ausstrichtechnik" in der übergroßen Zahl der Laboratorien aus wirtschaftlichen Gründen noch nicht eingesetzt werden kann. Wir suchten aber zunächst nach einem praxisnahen Vergleich.

2.1.2 Zum Vergleich wurden Ausstriche mikroskopiert, bei denen ein Blutstropfen auf einen Objektträger aufgebracht und kurz derartig zentrifugiert wurde, daß bei genau eingestellter Rotations- und Abbremsgeschwindigkeit („Spinner" von to spin = drehen, wirbeln) ein dünner Ausstrich entsteht, auf dem die Zellen gleichmäßig in einer Schicht verteilt sind und so nebeneinander liegen. Gefärbt wurde mit einem Färbeautomaten wiederum nach Wright.

Zur Auswertung der so angefertigten Ausstriche haben wir das Gerät "Microx 120" der Firma Omron', das nach dem Prinzip der Mustererkennung („pattern recognition") arbeitet, eingesetzt. Die gefärbten Ausstriche wer-

\footnotetext{
${ }^{1}$ Vertrieb Fa. Assista, Wien
}

den in Kassetten gestellt, aus denen sich die Maschine jeweils einen Ausstrich greift und dann - ohne daß jemand dabei sein muß - jeweils 300 Zellen differenziert. Die technischen Einzelheiten dieser Differenziermethode können hier übergangen werden. Wichtig ist, daß die Koordinaten jeder differenzierten Zelle gespeichert werden, so daß bei der Durchsicht der auf einem Bildschirm gezeigten Ergebnisse ("review") ein zweifelhafter Befund durch Aufruf jeder einzelnen Zelle als Bild auf dem Monitor noch einmal beurteilt werden kann. - Im Rahmen der hier geschilderten Untersuchungen wurden alle Monozyten und auch alle "unklassifizierbaren" Zellen auf diese Weise überprüft.

Die Verwendung des Microx 120 geschah nicht zum Zwecke der Vergleichbarkeit etwa mit dem VCS-System, sondern zur Optimierung - das heißt hier auch Vereinfachung - der Mikroskopie am Spinnerausstrich. Die Prüfung der Wertigkeit des "Pattern-recognition-Systems” hätte keinesfalls die optische Kontrolle jedes Monozyten und jeder unklassifizierten Zelle eingeschlossen.

Die Reproduzierbarkeit der Monozytenwerte bei diesem Verfahren wurde derart geprüft, daß die gleichen Ausstriche seitenvertauscht in die Kassette des Microx $120 \mathrm{ge}$ stellt wurden und auf diese Weise zweimal differenziert wurden $(n=29$ ). Die Regressionsgrade folgt der Gleichung Monozyten $1=0,933^{*}$ Monozyten $2+6,397, r=$ 0,891 .

2.1.3 Schließlich wurden mikroskopisch noch an jeweils 200 Zellen eines Ausstrichs die Zellen gezählt, die eine Positivität der $\alpha$-Naphthylacetatesterase zeigten. Hierbei handelt es sich um eine unspezifische Esterase, die bei der zytochemischen Differenzierung leukämischer Zellen als Marker der monozytären Differenzierung angesehen wird.

Die Untersuchungen wurden an frisch gewonnenem EDTA-Blut parallel innerhalb von maximal 90 Minuten durçhgeführt. Die luftgetrockneten Ausstriche wurden für 60 Minuten in folgendes Inkubationsgemisch gestellt: 4 Tropfen $4 \%$ Pararosanilin (gelöst in $2 \mathrm{~N} \mathrm{HCl}$ ) +4 Tropfen $4 \%$ Natriumnitrit (wäßrig), nach 60 Sekunden mit $40 \mathrm{ml}$ Phosphatpuffer $\mathrm{pH} 7$ vermischen, mit $\mathrm{KH}_{2} \mathrm{PO}_{4}$ auf $\mathrm{pH} 6,5$ einstellen. $\mathrm{Zu}$ dieser Lösung $10 \mathrm{mg} \alpha$-Naphthylacetat (gelöst in $1 \mathrm{ml}$ Aceton) geben und filtrieren. Abspülen in Aqua dest., Lufttrocknen, Gegenfärbung mit Hämalaun 5-10 Minuten in Leitungswasser bläuen, an der Luft trocknen.

Die Esterase-Reaktion wurde-erst während der Untersuchungsreihe zusätzlich eingeführt; sie berücksichtigt bei geringerer Probenzahl besonders höhere und niedrigere Normbereiche.

\subsection{Differenzierung mit der VCS-Technik}

2.2.1 Der Coulter ${ }^{\circledR}$ STKS differenziert im Durchflußverfahren die Zellen nach Volumen (V), innerer Leitfähigkeit (conductivity) und Streulichtverhalten nach Anregung mit einem Laserstrahl (scatter light). Auch hier soll auf technische Einzelheiten, die seit vielen Jahren bekannt sind, verzichtet werden. Differenziert wurden jeweils etwa 8000 Leukozyten.

2.2.2 Nach dem gleichen Prinzip arbeitet auch der jüngst auf den Markt gekommene Coulter MAXM, der eine vereinfachte Probenzuführung und eine leicht modifizierte Erkennungs- und Steuerungs-Software. (,research mode") hat. Der Zelldurchfluß beträgt wiederum etwas über 8000 Leukozyten. 
2.3. Differenzierung mittels markierter spezifischer Antikörper im Durchflußzytometer

Mittels der Durchflußfluorimetrie wurden mit dem Gerät EPICS Profile $\|^{2}$ an jeweils etwa 20000 Zellen die CD 14 (Markierung eines relativ spezifischen Monozytenantikörpers mit Phycoerythrin) und CD 45 (Pan-Leukozytenmarker, markiert mit FITC)-positiven Zellen gezählt (3).

Alle Untersuchungen wurden unmittelbar nach Eintreffen im Labor begonnen und innerhalb von vier Stunden für jeden Einzelfall vollständig abgeschlossen, wobei die Esterasefärbung einerseits, die zeitweilige Häufung des Probenanfalls andererseits die relativ lange Untersuchungsdauer erklären.

Alle Geräte wurden entsprechend den Original-Vorschriften der Hersteller eingesetzt.

Zum statistischen Vergleich wurde die standardisierte Hauptkomponentenanalyse eingesetzt (4), bei der eine Wertegruppe nicht vorgegeben, die andere damit verglichen wird, vielmehr beide Gruppen gleichwertig in die Rechnung eingehen.

\section{Ergebnisse}

Zunächst muß man von der oben geschilderten Annahme, der Handausstrich verteile die kernhaltigen Zellen immer so, daß die größeren Zellen an den Rand verschoben werden, abrücken. Beim Vergleich der Ergebnisse der Handausstriche mit der Differenzierung am Microx 120, bei dem jeder Monozyt auf einem Spinnerausstrich angesehen wurde (Abb. 1), zeigt sich, daß bei einer relativ breiten Streuung die Steigung der Geraden deutlich unter 1 liegt, die Monozytenwerte - gezählt am Handausstrich - also eher höher als auf dem Spinner-Ausstrich liegen. Dieses Ergebnis zeigt auch die vergleichende Zählung von jeweils 400 Zellen am Hand- und Spinnerausstrich am Mikroskop an einer kleinen Probe (Abb. 2). Hier ist die Steigung zwar optimal, die Streuung aber nicht vertretbar.

Aus einer früheren Untersuchung von Deinhardt (4) geht hervor, daß beim Vergleich von je 200 gezählten Zellen durch zwei medizinisch-technische Assistentinnen an Spinner-Ausstrichen (Abb. 3 ) eine sehr viel bessere Korrelation der Monozytenzahlen erreicht wird. Bei diesem Vergleich wurden allerdings auch Zellen im quantitativ pathologischen Bereich erfaßt.

Eine morphologische Referenzmethode wäre danach die mikroskopische Differenzierung von Spinnerausstrichen, wobei hier nur der Vergleich von jeweils 200 Zellen geprüft wurde.

Eine relativ einfache Methode, die Monozyten im Ausstrich zu erkennen, ist die cytochemische Reaktion mit Darstellung der $\alpha$-Napthylacetatesterase. Die Schwierigkeit bei dieser Methode liegt darin, daß die rostbraune Farbe des Reaktionsproduktes im Einzelfall so schwach ausfallen kann, daß eine sichere Zuordnung nicht möglich ist.

Auf kompliziertere, jedoch eindeutigere Weise lassen sich Monozyten mit der Durchflußcytometrie am Epics Profile II mit monoclonalen Antikörper nachweisen.

${ }^{2}$ Coulter Electronics, Krefeld

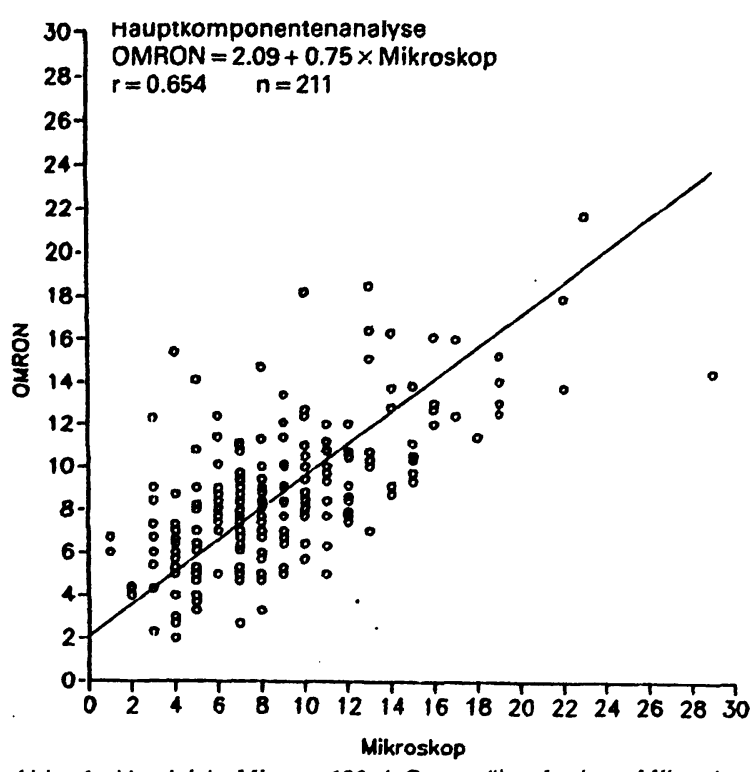

Abb. 1: Vergleich Microx 120 ("Omron“) mit dem Mikroskop (Handausstrich)

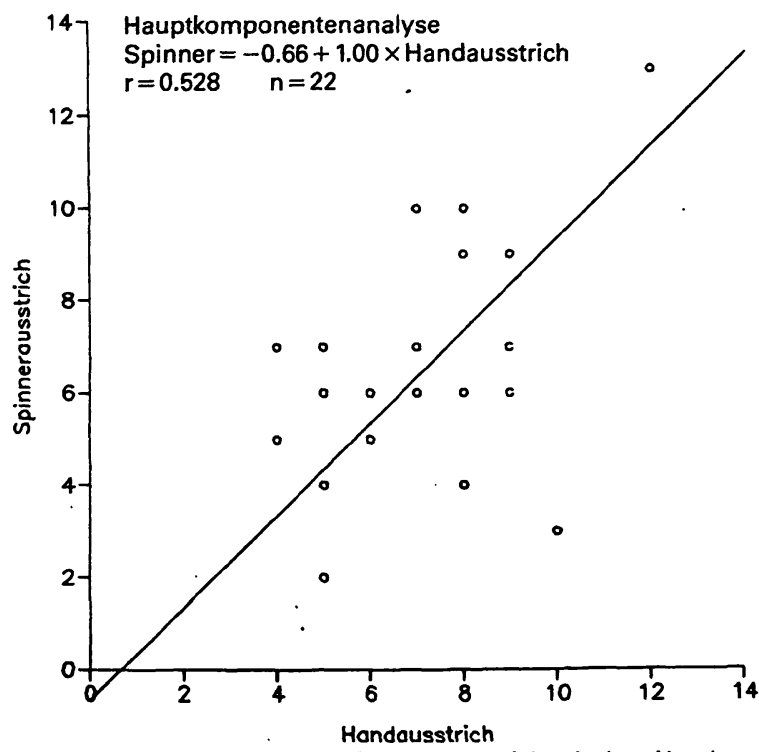

Abb. 2: Vergleich Mikroskop Spinnerausstrich mit dem Handausstrich

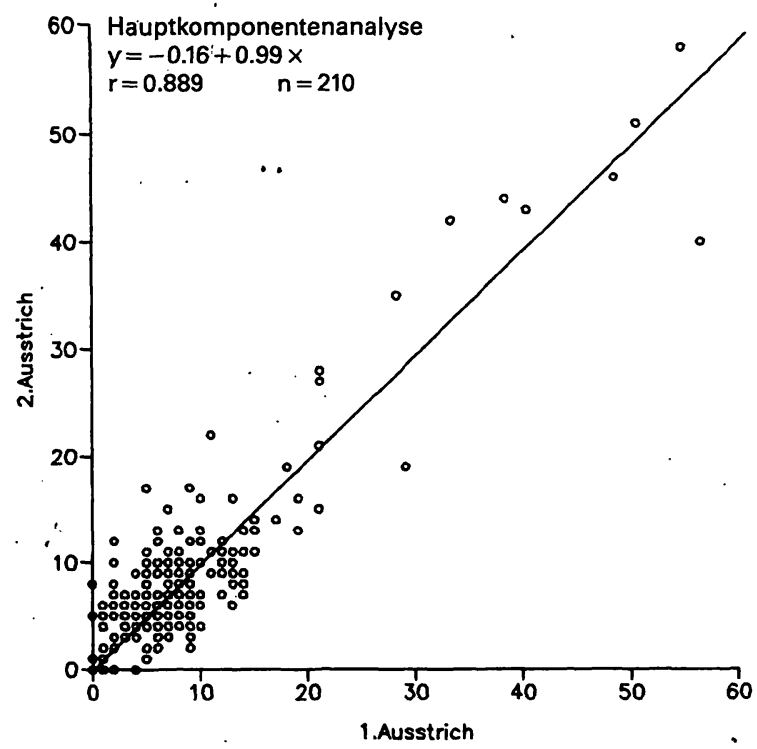

Abb. 3: Vergleich einer Differenzierung der gleichen Spinnerausstriche bei Differenzierung durch zwei technische Assistentinnen $(n=128)$ 


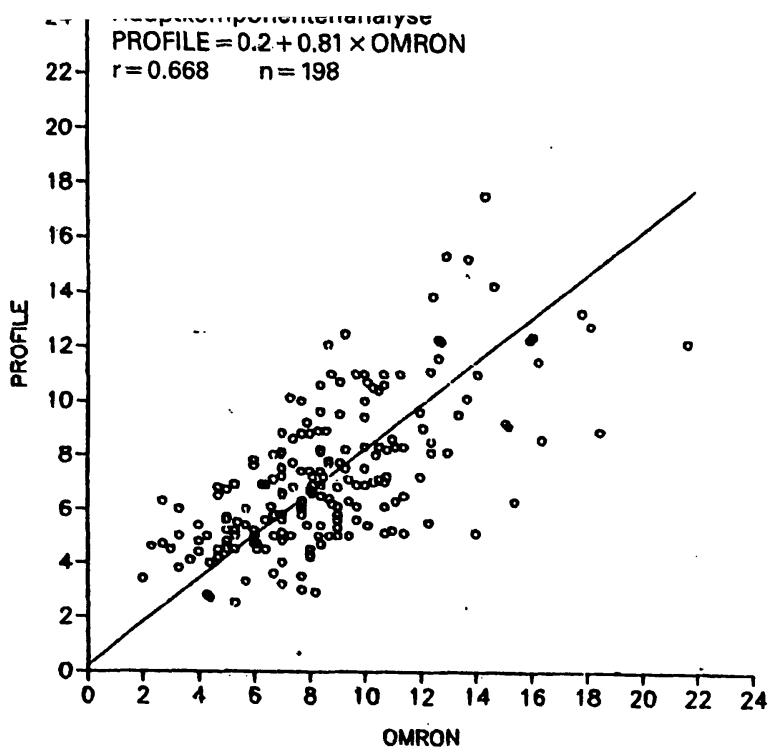

Abb. 4: Vergleich EPICS Profile II mit Microx 120

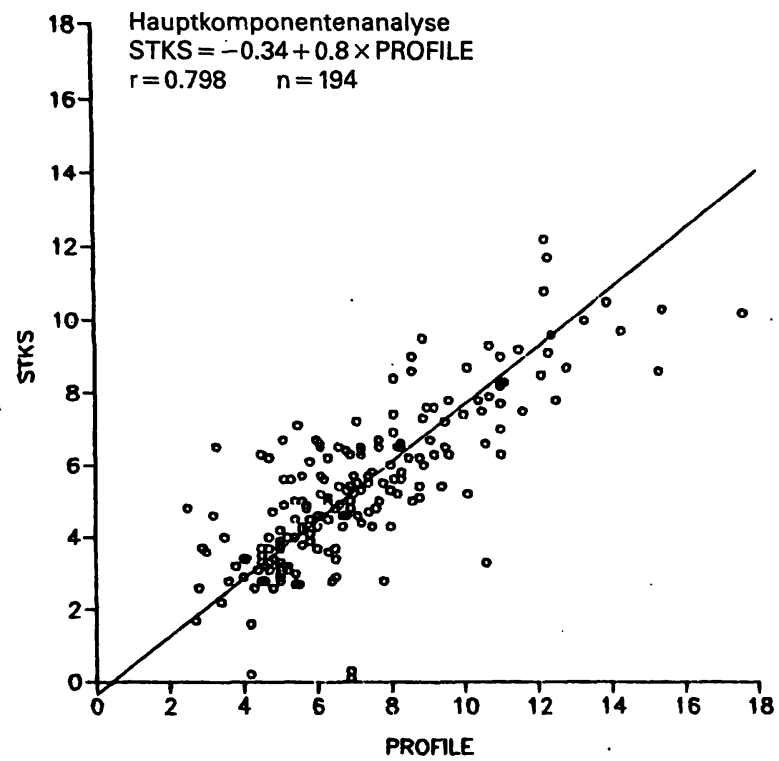

Abb. 6: Vergleich Ergebnisse-des Coulter STKS mit den Ergebnissen des EPICS Profile II

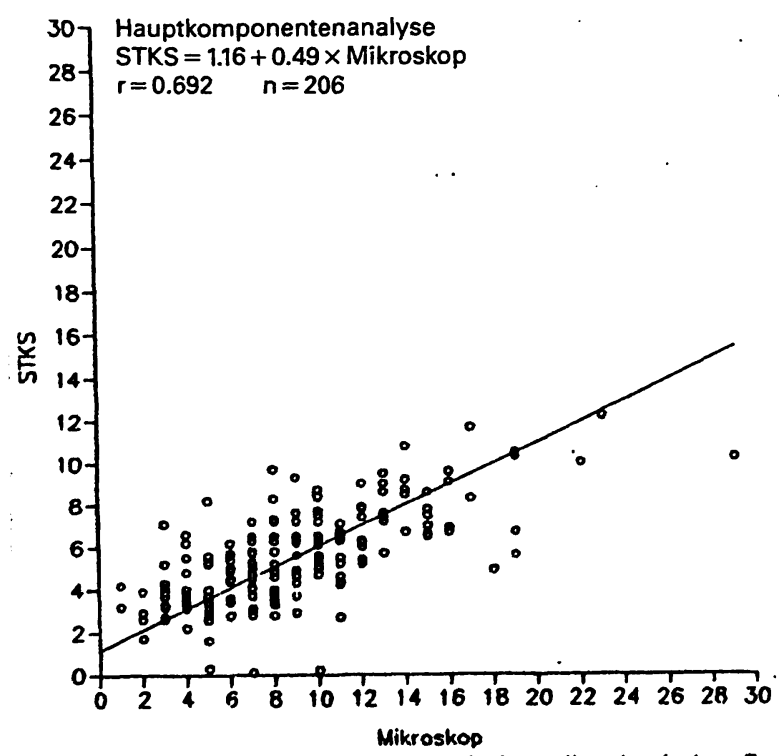

Abb. 8: Vergleich des Coulter STKS mit den mikroskoplschen Ergebnissen (Handausstrich)

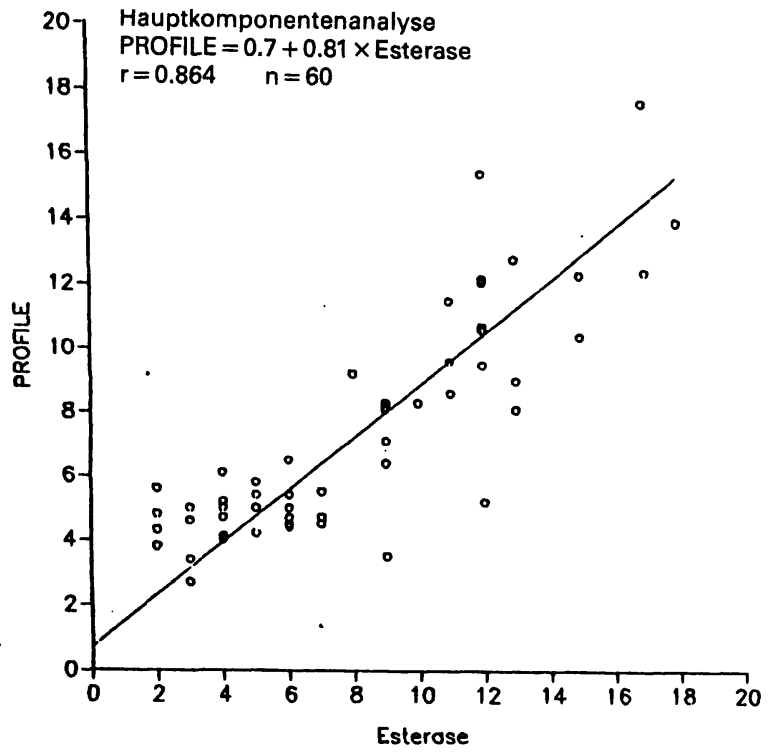

Abb. 5: Vergleich der mit $\alpha$-Naphthylacetat-Esterase positiven ZelIen (Spinnerausstrich) mit den Ergebnissen des EPICS Profile II

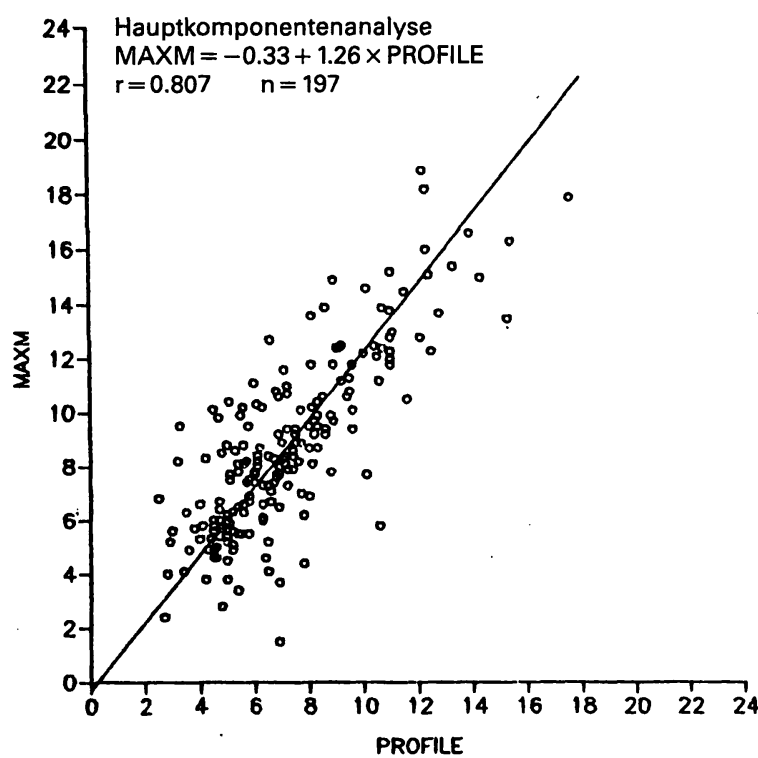

Abb. 7: Vergleich der Ergebnisse des Coulter MAXM mit denen des EPICS Profile

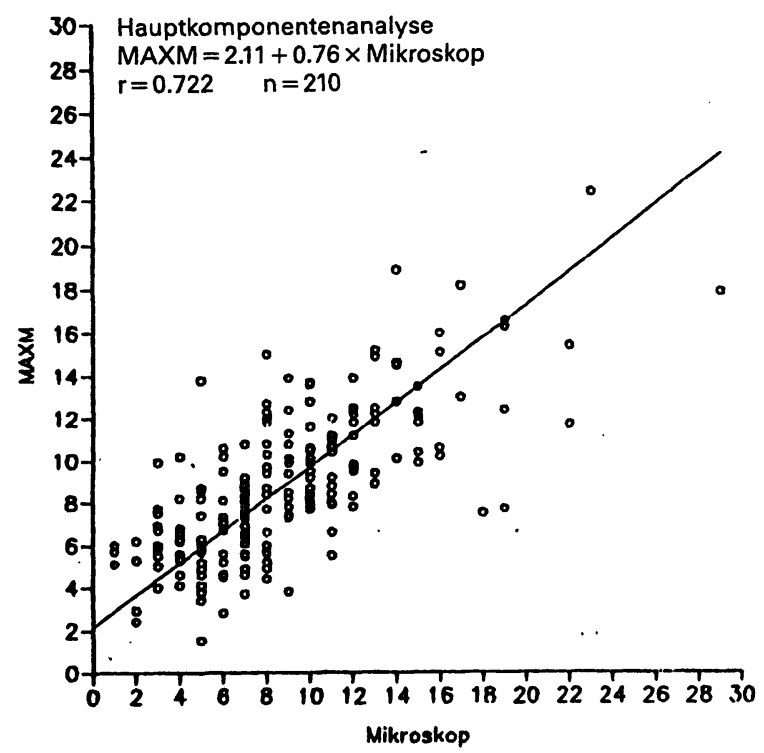

Abb. 9: Vergleich der Ergebnisse des Coulter MAXM mit dem mikroskopischen Ergebnis (Handausstrich) 
Der Vergleich der Monozytenzahlen von Microx 120, der die Mikroskopierung der Spinnerausstriche repräsentiert (Abb. 4), mit der Zahl der CD-14-markierten Zellen am Profile zeigt eine deutliche Streuung der Werte besonders im oberen Zählbereich, während Esterase-positive Zellen, die allerdings im mittleren Wertebereich nicht gut repräsentiert sind, bei gleicher Steigerung der Regressionsgraden mit den Werten des Omron Microx 120 besser korrelieren (Abb. 5).

Recht gut stimmen die Werte beim Vergleich des EPICS Profile II mit dem Coulter STKS, noch besser mit. dem Coulter MAX überein (Abb. 6 und 7).

Geht man von den mikroskopierten Handausstrichen aus, so ist die Übereinstimmung mit dem STKS, MAXM, der Esterase-Reaktion und dem Profile nicht befriedigend (Abb. 8-11).

Die Übereinstimmung zwischen Microx 120 und MAXM ist - gemessen an der Steigung der Regressionsgeraden - deutlich besser (Abb. 12).

\section{Diskussion}

Vergleichende Untersuchungen zwischen Differenzierungsautomaten unterschiedlicher Hersteller wurden naturgemäß häufiger durchgeführt. Sie alle hier zu würdigen, wäre für den Kundigen eine Zumutung. Zu dem hier erörterten Problem haben jüngst Goossens et al. (5) aufgrund eigener Untersuchungen Stellung genommen. Sie sahen die engsten Beziehungen zu den Ergebnissen der von ihnen angewendeten standardisierten Mikroskopiertechnik zur Durchflußcytometrie nach Doppelmarkierung $^{3}$. Beide Verfahren zeigten auch eine gute Reproduzierbarkeit der Werte bei der Wiederholung von jeweils 30 Analysen.

Bei den eigenen Untersuchungen zeigt sich, daß der Handausstrich für das Mikroskopieren zu einer sehr wechselnden Verteilung der kernhaltigen Zellen fühit, so daß diese Methode nicht als Vergleichsstandard gelten kann. Zuverlässigere Monozytenwerte sind an SpinnerAusstrichen zu gewinnen.

Ziel der vorliegenden Untersuchung war es, den Grad der Übereinstimmung zu prüfen, mit dem der neu eingeführte MAXM der Firma Coulter im Vergleich zur Mikroskopischen Differenzierung erreicht.

Nachdem der Handausstrich für das Mikroskop, der mit Rücksichtnahme auf die landläufige Praxis in den Labors noch immer als Maßstab angesehen wird, nicht als Standard gelten kann, war an Spinner-Ausstriche bei der Auswertung der. Untersuchung nur über den Microx $120 \mathrm{zu}$ kommen, der jeweils 300 Zellen differenziert, von denen alle gefundenen Monocyten visuell überprüft wurden.

Wenn man - der historischen Entwicklung folgend - davon ausgeht, daß die mikroskopische Untersuchung eines Spinner-Ausstrichs mit Differenzierung von $200 \mathrm{Zel}$ len (um im praktikablen Bereich zu verbleiben) als Bezugs-Standard gilt, muß der neue MAXM in unserem Fall mit den Ergebnissen des Microx 120 verglichen werden. Im Vergleich zu anderen Methoden, die aufwendiger sind (Esterase, Epics), ist die Korrelation vertretbar.

Nach der Entwicklung der letzten Jahre, die eine zunehmende Standardisierung der monoklonalen. Antikörper

\footnotetext{
${ }^{3}$ Hier mit dem FACScan der Fa. Becton Dickinson

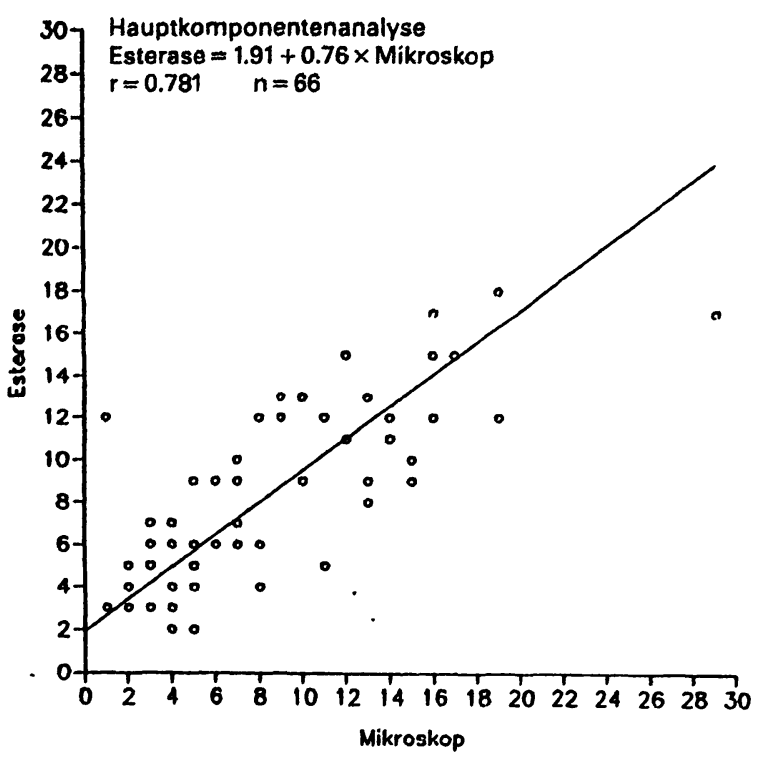

Abb. 10: Vergleich der Esterase-positiven Zellen (Spinnerausstrich) mit dem mikroskopisch differenzierten Handausstrich

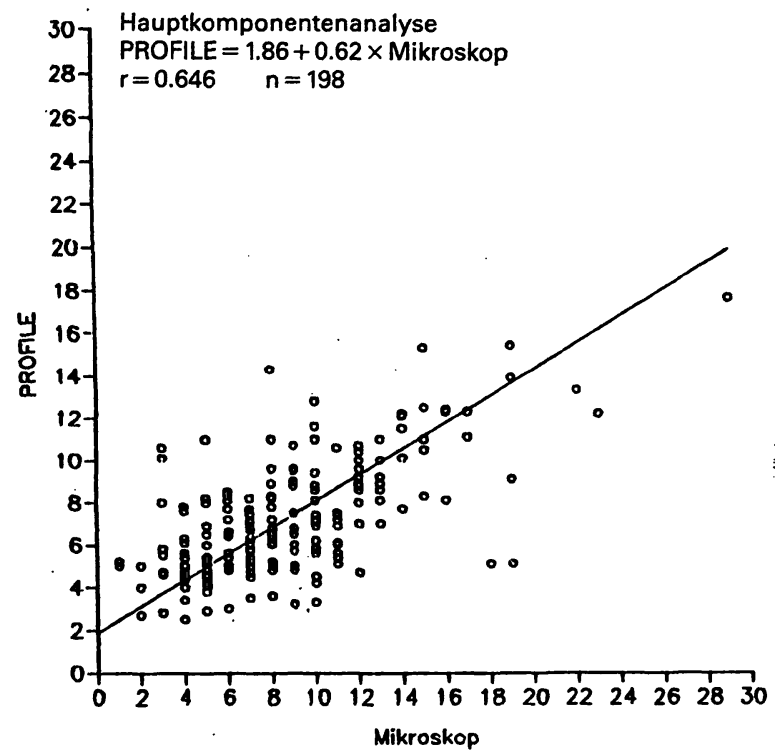

Abb. 11: Vergleich Profile mit dem mikroskopierten Handausstrich

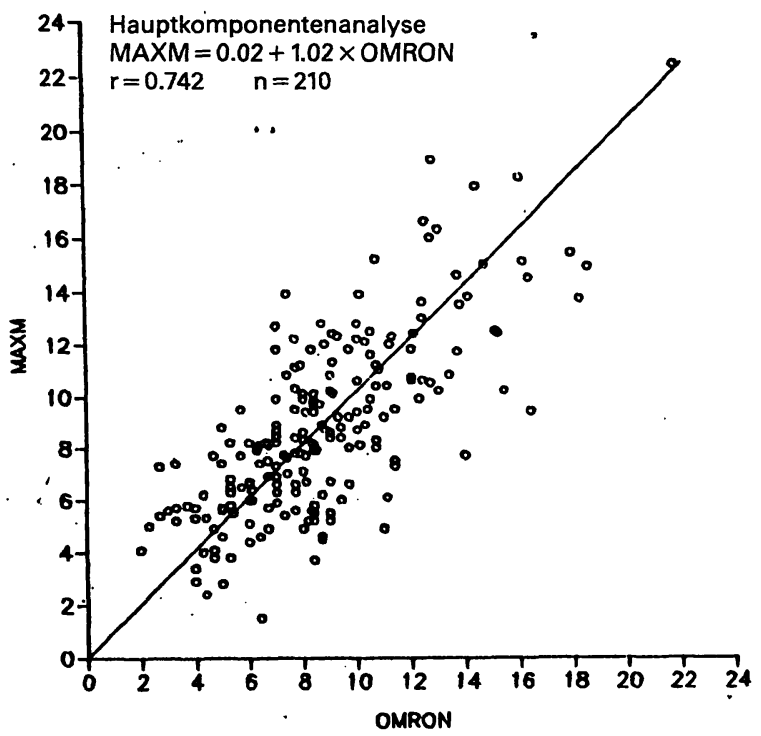

Abb. 12: Vergleich der Ergebnisse des Coulter MAXM mit den Ergebnissen des Microx 120 
gebracht haben, könnte die durchflußfluorimetrische Messung mit markierten Antikörpern zum Bezugsstandard für andere Geräte werden. Dieses Verfahren selbst wird in absehbarer Zeit nur in spezialisierten Laboratorien für besondere Fragestellungen, deren Lösung allein diese Methode erlaubt, Verwendung finden, nicht aber bei der Bewältigung der Alltagsroutine. - Sehr wohl aber kann es im Vergleich die Möglichkeiten der z. B. mit der VCS-Technik arbeitenden Geräte prüfen.

Auch wenn die Geräte mit der VCS-Technik, gemessen an der Immunfluoreszenztechnik, die Monozyten gut erkennen, setzt ein relativ hoher Probenanfall aus wirtschaftlichen Erwägungen den Einsatz derartiger Geräte voraus. Im weiten Feld der alltäglichen Praxis wird das Mikroskopieren (wohl auch an Handausstrichen) weiter verbreitet bleiben, sofern man nicht die Risiken, die ein Versand der Proben mit sich bringt, eingehen will. Hier ist es dann wichtig zu wissen, in welchem Sicherheitsrahmen man sich mit der optischen Differenzierung unter Berücksichtigung von Übung und Erfahrung bewegt.

Schließlich aber muß sich der Arzt, der aus den Laborwerten im Hinblick auf seinen Patienten Folgerungen ziehen will, darüber im klaren sein, daß die Kenntnis von Referenzwerten zwar unabdingbar ist, Konsequenzen bei geringen Über- oder Unterschreitungen sich aber nur unter Berücksichtigung des klinischen Gesamtbefundes ergeben dürfen. Für die Monozyten bedürfen deutliche Überschreitungen der oberen Normgrenze einer visuellen Kontrolle, da hier - bei Wahrung der nichtoptischen Kriterien der Monozyten - die Möglichkeit einer malignen Entartung dieser Zellen besteht.
Schrlfttum:

1. Begemann, H., Rastetter, J. (1987) „Atlas der klinischen Hämatologie”. Springer-Verlag, Berlin - Heidelberg-New York, 4. Aufl.

2. Klement, V. (1979) Über die semantisch gestützte maschinelle Analyse bildhafter Information am Beispiel mikroskopischer Zellbilder". Diss. TU Hannover.

3. Eckhardt, R. (1991) Durchflußzytometrie, eine schnelle und einfache Methode zur 3. Eckhardt, R. (1991) "Durchflußzytometrie, eine
Analyse großer Zellzahlen“. Lab.med 15, 563-569.

4. Feldmann, U., Schneider, B., Klinkers, H., Haeckel, R. (1981) „A Multivariate Approach for the Biometric Comparison of Analytical Methods in Clinical Chemistry". J. Clin. Chem. Clin. Biochem. 19, 121-137.

5. Deinhardt, J. „Durchflußzytometer für die Hămatologie von COULTER” (unveröffentlicht).

6. Goossens, W., Van Hove, L. Verwilghen, R. L. (1991) „Monocyte counting: Discrepancies in results obtained with different automated instruments". J. Clin. Pathol. 44 224-227.

\section{Danksagung}

Wir danken den Damen U. Bormann-Danso, S. Grell, C. Kemmerling, H. Röhlinger, den Herren R. Kirstein und D. Klose für ihre sorgfältige Mitarbeit. - Herrn Dipl.-Math. H. Geerlings (Abt. Biometrie der $\mathrm{MHH}$ ) danken wir für fachspezifische Beratung und die Durchführung von Vergleichstests.

\section{Anschrift der Verfasser:}

Prof. Dr. med. Hans Jürgen Avenarius

Dipl.-Phys. Joachim Deinhardt

Medizinische Hochschule Hannover

Abt. Hämatologie-Onkologie

Konstanty-Gutschow-Straße 8

3000 Hannover 61 